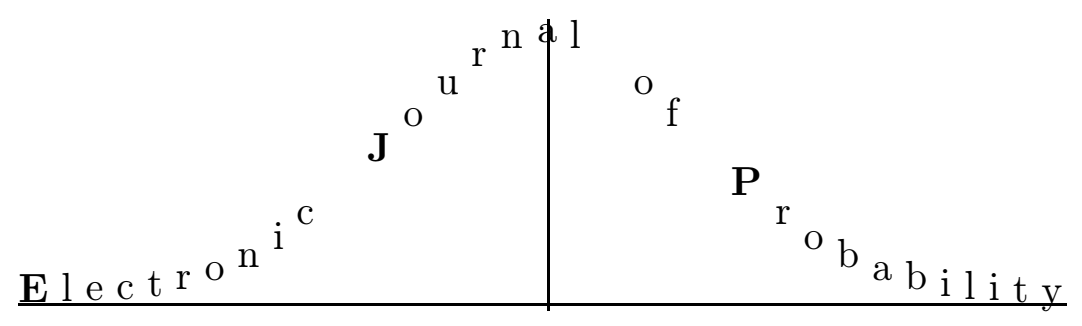

Vol. 2 (1997) Paper no. 4, pages 1-21.

Journal URL

http://www.math.washington.edu/ ejpecp/

Paper URL

http://www.math.washington.edu/ ejpecp/EjpVol2/paper4.abs.html

\title{
A NON-SKOROHOD TOPOLOGY ON THE SKOROHOD SPACE
}

\author{
Adam Jakubowski \\ Nicholas Copernicus University \\ Faculty of Mathematics and Computer Science \\ ul. Chopina 12/18, 87-100 Toruń, Poland \\ E-mail: adjakubo@mat.uni.torun.pl
}

\begin{abstract}
A new topology (called $S$ ) is defined on the space $\mathbb{D}$ of functions $x:[0,1] \rightarrow \mathbb{R}^{1}$ which are right-continuous and admit limits from the left at each $t>0$. Although $S$ cannot be metricized, it is quite natural and shares many useful properties with the traditional Skorohod's topologies $J_{1}$ and $M_{1}$. In particular, on the space $\mathcal{P}(\mathbb{D})$ of laws of stochastic processes with trajectories in $\mathbb{D}$ the topology $S$ induces a sequential topology for which both the direct and the converse Prohorov's theorems are valid, the a.s. Skorohod representation for subsequences exists and finite dimensional convergence outside a countable set holds.
\end{abstract}

Keywords: Skorohod space, Skorohod representation, convergence in distribution, sequential spaces, semimartingales.

AMS subject classification: 60F17, 60B05, 60G17, 54D55.

Research supported by Komitet Badań Naukowych under Grant No 211089101.

Submitted to EJP on April 1, 1996. Final version accepted on July 4, 1997. 


\title{
A non-Skorohod topology on the Skorohod space
}

\author{
Adam Jakubowski* \\ Nicholas Copernicus University, Toruń, Poland
}

\begin{abstract}
A new topology (called $S$ ) is defined on the space $\mathbb{D}$ of functions $x:[0,1] \rightarrow \mathbb{R}^{1}$ which are right-continuous and admit limits from the left at each $t>0$. Although $S$ cannot be metricized, it is quite natural and shares many useful properties with the traditional Skorohod's topologies $J_{1}$ and $M_{1}$. In particular, on the space $\mathcal{P}(\mathbb{D})$ of laws of stochastic processes with trajectories in $\mathbb{D}$ the topology $S$ induces a sequential topology for which both the direct and the converse Prohorov's theorems are valid, the a.s. Skorohod representation for subsequences exists and finite dimensional convergence outside a countable set holds.
\end{abstract}

\section{Introduction}

Let $\mathbb{D}=\mathbb{D}\left([0,1]: \mathbb{R}^{1}\right)$ be the space of functions $x:[0,1] \rightarrow \mathbb{R}^{1}$ which are right-continuous and admit limits from the left at each $t>0$. We are going to study a new sequential topology on $\mathbb{D}$ generated by naturally arising criteria of relative compactness. The novelty is that this topology cannot be metricized. Nevertheless we shall show how to build a complete and satisfactory theory of the convergence in distribution with respect to this topology.

Despite the fact that metric topologies are sequential, the process of defining topology through description of the family of convergent sequences is not the common approach, especially in probability theory. We refer to [7] for rather extensive discussion of sequential methods in weak limit theorems of probability theory. Here we shall point out only that

every time one proves a limit theorem via the direct Prohorov's theorem, one obtains a result for the sequential topology generated by the original one.

Since Prohorov's theorems are accepted tools of probability theory, the same should happen to sequential methods, as they fit the original ideas of Prohorov and Skorohod much better than the theory based on weak-* convergence of distributions.

In order to show our motivations we begin with a simple, well-known example. Let $\mathbb{V}^{+} \subset \mathbb{D}$ consists of nonnegative and nondecreasing functions $v:[0,1] \rightarrow \mathbb{R}^{+}$. Suppose that for some subset $K \subset \mathbb{V}^{+}$we have:

$$
\sup _{v \in K} v(1)<+\infty .
$$

* Research supported by Komitet Badań Naukowych under Grant No 211089101 
Let $\mathbb{Q} \subset[0,1]$ be countable dense and let $1 \in \mathbb{Q}$. By (1) we may find a sequence $\left\{v_{n}\right\}_{n \in \mathbb{N}} \subset K$ such that for each $q \in \mathbb{Q}$

$$
v_{n}(q) \rightarrow \tilde{v}(q)
$$

where $\tilde{v}(q)$ are numbers satisfying $\tilde{v}\left(q^{\prime}\right) \leq \tilde{v}\left(q^{\prime \prime}\right), q^{\prime}<q^{\prime \prime}, q^{\prime}, q^{\prime \prime} \in \mathbb{Q}$. Hence the function

$$
v_{0}(t)= \begin{cases}\inf _{\substack{q>t \\ q \in \mathbb{Q}}} \tilde{v}(q) & \text { if } t<1 \\ \tilde{v}(1) & \text { if } t=1\end{cases}
$$

belongs to $\mathbb{V}^{+}$. Moreover, we have as $n \rightarrow \infty$

$$
v_{n}(t) \rightarrow v_{0}(t)
$$

provided $t=1$ or $t$ is a point of continuity of $v_{0}: v_{0}(t)=v_{0}(t-)$. Define finite measures on $\left([0,1], \mathcal{B}_{[0,1]}\right)$ by the formula

$$
\mu_{n}([0, t])=v_{n}(t), t \in[0,1], n=0,1,2, \ldots,
$$

and observe that (3) is equivalent to the weak convergence of $\mu_{n}$ 's, i.e. convergence of $\mu_{n}$ 's considered as continuous linear functionals on the space $C\left([0,1]: \mathbb{R}^{1}\right)$ of continuous functions on $[0,1]$ equipped with the weak-* topology:

$$
\mu_{n} \Rightarrow \mu_{0} \quad \text { iff } \quad \int f(t) d \mu_{n}(t) \rightarrow \int f(t) d \mu_{0}(t), f \in C\left([0,1]: \mathbb{R}^{1}\right) .
$$

It follows that condition (1) when restricted to $\mathbb{V}^{+}$is a criterion of relative compactness for some, quite natural topology.

A very similar procedure may be performed for the space $\mathbb{D}$. Suppose that

$$
\sup _{x \in K} \sup _{t \in[0,1]}|x(t)| \leq C_{K}<+\infty
$$

and that for all $a<b, a, b \in \mathbb{R}$

$$
\sup _{x \in K} N^{a, b}(x) \leq C_{K}^{a, b}<+\infty
$$

where $N^{a, b}$ is the usual number of up-crossing given levels $a<b$. (Recall that $N^{a, b}(x) \geq k$ if one can find numbers $0 \leq t_{1}<t_{2}<\ldots<t_{2 k-1}<t_{2 k} \leq 1$ such that $x\left(t_{2 i-1}\right)<a$ and $x\left(t_{2 i}\right)>b$, $i=1,2, \ldots, k)$. Let, as previously, $\mathbb{Q} \subset[0,1], \mathbb{Q} \ni 1$, be countable dense. By (4) we can extract a sequence $\left\{x_{n}\right\}_{n \in \mathbb{N}} \subset K$ such that, as $n \rightarrow \infty$

$$
x_{n}(q) \rightarrow \tilde{x}(q), q \in \mathbb{Q} .
$$

Now the construction of the limiting function $x_{0}$ is not as easy as in the case of $\mathbb{V}^{+}$and one has to use (5) in an essential way to see that

$$
x_{0}(t)= \begin{cases}\lim _{\substack{q \rightarrow t+\\ q \in \mathbb{Q}}} \tilde{x}(q) & \text { if } t<1 \\ \tilde{x}(1) & \text { if } \quad t=1\end{cases}
$$


is well-defined and belongs to $\mathbb{D}$. And whether $x_{n}$ converges to $x_{0}$ in some topology on $\mathbb{D}$ is not clear at all.

Meyer and Zheng [14] considered on $\mathbb{D}$ so-called pseudo-path topology and proved that (4) and (5) imply conditional compactness of $K$ in this topology. However, the pseudo-path topology was shown to be equivalent on $\mathbb{D}$ to the convergence in (Lebesgue) measure. And so neither (4) nor (5) form the proper description of the relative compactness in the pseudo-path topology, for it is easy to find a sequence $\left\{x_{n}\right\}$ of elements of $\mathbb{D}$ which is convergent in measure to an element $x_{0} \in \mathbb{D}$ and is such that for $K=\left\{x_{n}: n=0,1,2, \ldots\right\}$ both (4) and (5) are not satisfied.

In the present paper we construct a topology on $\mathbb{D}$, say $S$, which possesses the following properties.

- $K \subset \mathbb{D}$ is relatively $S$-compact iff both (4) and (5) hold.

- $S$ is sequential and cannot be metricized.

- There exists a countable family of $S$-continuous functions which separate points in $\mathbb{D}$.

- The $\sigma$-field $\mathcal{B}_{S}$ of Borel subsets for $S$ coincides with the usual $\sigma$-field generated by projections (or evaluations) on $\mathbb{D}: \mathcal{B}_{S}=\sigma\left(\pi_{t}: t \in[0,1]\right)$.

- The set $\mathcal{P}(\mathbb{D}, S)$ of $S$-tight probability measures is exactly the set of distributions of stochastic processes with trajectories in $\mathbb{D}: \mathcal{P}(\mathbb{D}, S)=\mathcal{P}(\mathbb{D})$.

- $S$ is weaker than Skorohod's $M_{1}$ and $J_{1}$ topologies. Since $J_{1}$ is Polish, $S$ is Lusin in the sense of Fernique. But we do not know whether it is (completely) regular.

In the absence of regularity almost nothing of the theory for Lusin spaces (as described in [3] and [4]) can be used. There exists, however, a different approach, developed in [7] and based on the following strong form of the direct Prohorov's theorem (see [5, Theorem 2]):

Theorem 1.1 Let $(\mathcal{X}, \tau)$ be a topological space on which there exists a countable family of $\tau$ continuous functions separating points in $X$. Let $\left\{\mu_{n}\right\}_{n \in \mathbb{N}}$ be a uniformly tight sequence of laws on $\mathcal{X}$. Then there exists a subsequence $n_{1}<n_{2}<\ldots$ and $\mathcal{X}$-valued random elements $Y_{0}, Y_{1}, Y_{2}, \ldots$ defined on $\left([0,1], \mathcal{B}_{[0,1]}, \ell\right)$ such that

$$
\begin{gathered}
X_{n_{k}} \sim Y_{k}, \quad k=1,2, \ldots, \\
Y_{k}(\omega) \underset{\tau}{\longrightarrow} Y_{0}(\omega), \quad \text { as } k \rightarrow \infty, \omega \in[0,1] .
\end{gathered}
$$

It is clear that our space $(\mathbb{D}, S)$ fulfills the only assumption of the above theorem and so in the space $\mathcal{P}(\mathbb{D}, S)$ the direct Prohorov's theorem is valid. Notice that we are also very close to the a.s. Skorohod representation.

One can go further in this direction and following [7] define a new sequential topology $\mathcal{O}(\stackrel{*}{\Longrightarrow})$ (where $\stackrel{*}{\Longrightarrow}$ denotes the convergence determining the topology) on $\mathcal{P}(\mathbb{D}, S)$ with the following properties:

- $\mathcal{O}(\stackrel{*}{\Longrightarrow})$ is the unique sequential topology on $\mathcal{P}(\mathbb{D})$ which is finer than the weak topology and for which $\stackrel{*}{\Longrightarrow}$-relative compactness coincides with uniform $S$-tightness. In particular, for $\mathcal{O}(\stackrel{*}{\Longrightarrow})$ both the direct and the converse Prohorov's theorems are valid. 
- Let $X_{n} \stackrel{*^{*}}{\longrightarrow} \mathcal{D} X_{0}$ mean that the laws of processes $X_{n}$ converge in the new sense: $\mathcal{L}\left(X_{n}\right) \stackrel{*}{\Longrightarrow}$ $\mathcal{L}\left(X_{0}\right)$. Suppose $X_{n} \stackrel{*^{\prime}}{\longrightarrow} \mathcal{D} X_{0}$. Then in each subsequence $\left\{X_{n_{k}}\right\}_{k \in \mathbb{N}}$ one can find a further subsequence $\left\{X_{n_{k_{l}}}\right\}_{l \in \mathbb{N}}$ such that:

- $\left\{X_{0}\right\} \cup\left\{X_{n_{k_{l}}}: l=1,2, \ldots\right\}$ admit the usual a.s. Skorohod representation on $\left([0,1], \mathcal{B}_{[0,1]}\right)$;

- outside some countable set $\mathbb{Q}_{0} \subset[0,1)$ all finite dimensional distributions of $\left\{X_{n_{k_{l}}}\right\}$ converge to those of $X_{0}$.

The last statement improves a corresponding result due to Meyer and Zheng [14], where finite dimensional convergence outside a set of Lebesgue measure null was shown via results on the pseudo-path topology.

Results on finite dimensional convergence are of quite different flavour than in the case of Skorohod's metric topologies $M_{1}$ and $J_{1}$ : for every $t \neq 1$ the projection $\pi_{t}: \mathbb{D} \rightarrow \mathbb{R}^{1}, \pi_{t}(x)=x(t)$, is nowhere $S$-continuous and so the standard continuity arguments cannot be applied. There exists, however, a simple procedure, completely paralleling the one described for $\mathbb{D}$ in (4) - (7) and allowing to determine the finite dimensional distributions of the limiting process (hence identifying the limits). Suppose that $\left\{X_{n}\right\}$ is a uniformly $S$-tight sequence. Choose a dense countable subset $\mathbb{Q} \subset[0,1], \mathbb{Q} \ni 1$, and extract a subsequence $\left\{X_{n_{k}}\right\}$ such that for each finite sequence $q_{1}<q_{2}<$ $\ldots<q_{m}$ of elements of $\mathbb{Q}$ we have

$$
\left(X_{n_{k}}\left(q_{1}\right), X_{n_{k}}\left(q_{2}\right), \ldots, X_{n_{k}}\left(q_{m}\right)\right) \underset{\mathcal{D}}{\longrightarrow} \tilde{\nu}_{\left(q_{1}, q_{2}, \ldots, q_{m}\right)},
$$

where $\tilde{\nu}_{\left(q_{1}, q_{2}, \ldots, q_{m}\right)}$ is a probability distribution on $\mathbb{R}^{m}$. Then one can prove that for each finite sequence $t_{1}<t_{2}<\ldots<t_{m}$ and each approximating sequence $q_{1, l}<q_{2, l}<\ldots<q_{m, l}, q_{j, l} \searrow t_{j}$, as $l \rightarrow \infty,\left(q_{m, l}=1\right.$ if $\left.t_{m}=1\right)$, probability distributions $\tilde{\nu}_{\left(q_{1, l}, q_{2, l}, \ldots, q_{m, l}\right)}$ weakly converge to some limit $\nu_{\left(t_{1}, t_{2}, \ldots, t_{m}\right)}$. Moreover, there is a stochastic process $X_{0}$ with trajectories in $\mathbb{D}$ such that for every finite sequence $t_{1}<t_{2}<\ldots<t_{m}$

$$
\left(X_{0}\left(t_{1}\right), X_{0}\left(t_{2}\right), \ldots, X_{0}\left(t_{m}\right)\right) \sim \nu_{\left(t_{1}, t_{2}, \ldots, t_{m}\right)},
$$

and $X_{n_{k}} \stackrel{*}{\longrightarrow} \mathcal{D} \quad X_{0}$. This simple procedure was known to hold in the Skorohod $J_{1}$-topology ([20]); it is interesting to see that it preserves validity at much lower level.

The paper is organized as follows.

In Section 2 we define the topology $S$ and give its basic properties.

In Section 3 we apply to the space $\mathcal{P}(\mathbb{D}, \mathcal{S})$ the machinery developed in [7] which is suitable for spaces with countable continuous separation property (as it is the case). In particular we prove all the results announced above.

In Section 4 we show how the theory can be applied to sets of semimartingales satisfying socalled Condition UT which implies uniform $S$-tightness and which is known to be important in limit theorems for stochastic integrals and stochastic differential equations ([8], [17],[12], [18], [6]).

\section{The topology $S$}

We shall define the topology $S$ in several steps, following the strategy for sequential topologies described in Section 3 of [7] (the reader is referred to that paper for definitions and notation). Here is the description of subsequent steps. 
1. Give equivalent reformulations for the (potential) criteria of compactness (4) and (5) - Lemma 2.1 .

2. Find an $\mathcal{L}$-convergence for which (4) and (5) are criteria of relative compactness-Lemma 2.7 .

3. Define $S$-topology and construct an $\mathcal{L}^{*}$-convergence using the Kantorovich-Kisyński recipe.

4. Show there exists a countable family of $S$-continuous functions separating points of $\mathbb{D}$ and conclude the topology $S$ is suitable for needs of probability theory.

5. Investigate in some detail other properties of the topology $S$ (Propositions 2.14 and 2.15).

Let us denote by $\|x\|_{\infty}$ the supremum norm on $\mathbb{D}$ :

$$
\|x\|_{\infty}=\sup _{t \in[0,1]}|x(t)|
$$

and by $\|v\|$ the total variation of $v$ :

$$
\|v\|(t)=\sup \left\{|v(0)|+\sum_{i=1}^{m}\left|v\left(t_{i}\right)-v\left(t_{i-1}\right)\right|: 0=t_{0}<t_{1}<\ldots<t_{m}=t, m \in \mathbb{N}\right\} .
$$

For $a<b$ let the number of up-crossings $N^{a, b}$ be defined as above (see the lines after formula (5)) and for $\eta>0$ let the number $N_{\eta}$ of $\eta$-oscillations be defined by the relation: $N_{\eta}(x) \geq k$ iff one can find numbers $0 \leq t_{1} \leq t_{2} \leq \ldots \leq t_{2 k-1} \leq t_{2 k} \leq 1$ such that $\left|x\left(t_{2 i-1}\right)-x\left(t_{2 i}\right)\right|>\eta, i=1,2, \ldots, k$. Finally, let $\mathbb{V}=\mathbb{V}^{+}-\mathbb{V}^{+}$.

Lemma 2.1 Let $K \subset \mathbb{D}$ and suppose that

$$
\sup _{x \in K}\|x\|_{\infty}<+\infty
$$

Then the statements (i) and (ii) below are equivalent:

(i) For each $a<b$

$$
\sup _{x \in K} N^{a, b}(x)<+\infty
$$

(ii) For each $\eta>0$

$$
\sup _{x \in K} N_{\eta}(x)<+\infty
$$

Moreover, either set of conditions (12) $+(13)$ and (12) $+(14)$ is equivalent to

(iii) For each $\varepsilon>0$ and for each $x \in K$ there exists $v_{x, \varepsilon} \in \mathbb{V}$ such that

$$
\sup _{x \in K}\left\|x-v_{x, \varepsilon}\right\|_{\infty} \leq \varepsilon,
$$

and

$$
\sup _{x \in K}\left\|v_{x, \varepsilon}\right\|(1)<+\infty
$$


Proof. Let us observe first that (iii) implies (12):

$$
\|x\|_{\infty} \leq \varepsilon+\left\|v_{x, \varepsilon}\right\|(1) .
$$

Then the chain of implications (iii) $\Rightarrow(\mathbf{i i}) \Rightarrow$ (i) follows by inequalities

$$
N_{\eta}(x) \leq \frac{\left\|v_{x, \varepsilon}\right\|(1)}{\eta-2 \varepsilon}, \quad \eta>2 \varepsilon>0, x \in \mathbb{D}
$$

and

$$
N^{a, b}(x) \leq N_{b-a}(x), \quad b>a, x \in \mathbb{D} .
$$

Now assume (12). First we shall prove (i) $\Rightarrow\left(\right.$ ii). Let $C_{\infty}=\sup _{x \in K}\|x\|_{\infty}$ and suppose that for some $\eta>0$ and every $n \in \mathbb{N}$ there is $x_{n} \in K$ such that $N_{\eta}\left(x_{n}\right) \geq n$. In particular, for some $0 \leq t_{n, 1}<t_{n, 2}<\ldots<t_{n, 2 n-1}<t_{n, 2 n} \leq 1$ we have $\left|x_{n}\left(t_{n, 2 i}\right)-x_{n}\left(t_{n, 2 i-1}\right)\right|>\eta, i=1,2, \ldots, n$. Let $a_{1}<a_{2}<\ldots<a_{R}$ be an $\eta / 2$-net for the interval $I=\left[-C_{\infty}, C_{\infty}\right]$, i.e. for each $x \in I$ there is $a_{j}$ such that $\left|x-a_{j}\right|<\eta / 2$. In every interval $I_{n, i}$ with ends in points $x_{n}\left(t_{n, 2 i-1}\right)$ and $x_{n}\left(t_{n, 2 i}\right)$ there are at least two points $a_{j-1}$ and $a_{j}$ which belong to $I_{n, i}$ and are distinct from the interval's ends. Let $M_{j}(n)$ be the number of $i$ 's such that both $a_{j-1}$ and $a_{j}$ belong to $I_{n, i}$. It follows that for some $j_{0}$

$$
\sup _{n} M_{j_{0}}\left(x_{n}\right)=+\infty
$$

But $N^{a_{j_{0}-1}, a_{j_{0}}}\left(x_{n}\right)$ is greater or equal to the integer part of $\left(M_{j_{0}}\left(x_{n}\right)-1\right) / 2$ and so by $(20)$

$$
\sup _{x \in K} N^{a_{j_{0}-1}, a_{j_{0}}}(x) \geq \sup _{n} N^{a_{j_{0}-1}, a_{j_{0}}}\left(x_{n}\right)=+\infty .
$$

It remains to prove (ii) $\Rightarrow\left(\right.$ iii). The construction of $v_{x, \varepsilon}$ is, in some sense, standard. For $\varepsilon>0$ let us define

$$
\begin{aligned}
\tau_{0}^{\varepsilon}(x) & =0 \\
\tau_{k}^{\varepsilon}(x) & =\inf \left\{t>\tau_{k-1}^{\varepsilon}(x):\left|x(t)-x\left(\tau_{k-1}^{\varepsilon}(x)\right)\right|>\varepsilon\right\}, k=1,2, \ldots
\end{aligned}
$$

(where by convention $\inf \emptyset=+\infty$ ) and let

$$
v_{\varepsilon}(x)(t)=x\left(\tau_{k}^{\varepsilon}(x)\right) \text { if } \tau_{k}^{\varepsilon}(x) \leq t<\tau_{k+1}^{\varepsilon}(x), t \in[0,1], k=0,1,2, \ldots
$$

Then by the very definition

$$
\begin{aligned}
\left\|x-v_{\varepsilon}(x)\right\|_{\infty} & \leq \varepsilon \\
\left\|v_{\varepsilon}(x)\right\|(1) & \leq\|x\|_{\infty}\left(2 N_{\varepsilon / 2}(x)+1\right)
\end{aligned}
$$

and the lemma follows.

Corollary 2.2 For each $t \in[0,1]$ the mapping $\mathbb{D} \ni x \mapsto\left(v_{\varepsilon}(x)\right)(t)$ defined by (23) is $\mathcal{F}_{t+-}$ measurable, where $\left\{\mathcal{F}_{t+}\right\}_{t \in[0,1]}$ is the natural right-continuous filtration on the canonical space $\mathbb{D}$ : $\mathcal{F}_{t+}=\cap_{u>t} \sigma\left(\pi_{s}: 0 \leq s \leq u\right)$. Hence $v_{\varepsilon}(X)$ is an adapted stochastic process provided $X$ is adapted to a right-continuous filtration. 
Before introducing a convergence in $\mathbb{D}$ which generates the $S$-topology, let us recall some facts on the weak- $*$ topology on $\mathbb{V}$. Any element $v \in \mathbb{V}$ determines a signed measure $\nu$ on $([0,1], \mathcal{B})$ given by the formula

$$
\nu([0, t])=v(t), \quad t \in[0,1] .
$$

Since the set of signed measures can be identified with the dual of the Banach space $C\left([0,1]: \mathbb{R}^{1}\right)$, $\mathbb{V}$ can be equipped with the weak-* topology. Convergence of elements of $\mathbb{V}$ in this topology will be denoted by $\longrightarrow_{w}$. In particular, $v_{n} \longrightarrow_{w} v_{0}$ means that for every continuous function $f:[0,1] \rightarrow \mathbb{R}^{1}$

$$
\int_{[0,1]} f(t) d v_{n}(t) \underset{w}{\longrightarrow} \int_{[0,1]} f(t) d v_{0}(t) .
$$

Definition 2.3 We shall write $x_{n} \longrightarrow_{S} x_{0}$ if for every $\varepsilon>0$ one can find elements $v_{n, \varepsilon}, n=$ $0,1,2, \ldots$ which are $\varepsilon$-uniformly close to $x_{n}$ 's and weakly-* convergent:

$$
\begin{array}{r}
\left\|x_{n}-v_{n, \varepsilon}\right\|_{\infty} \leq \varepsilon, \quad n=0,1,2, \ldots, \\
v_{n, \varepsilon} \underset{w}{\longrightarrow} v_{0, \varepsilon}, \quad \text { as } n \rightarrow+\infty .
\end{array}
$$

Remark 2.4 (28) implies that

$$
v_{n, \varepsilon}(t) \rightarrow v_{0, \varepsilon}(t)
$$

for each $t$ outside a countable set $D_{\varepsilon} \subset[0,1)$. Taking $\varepsilon=1,1 / 2,1 / 3, \ldots$ we obtain

$$
x_{n}(t) \rightarrow x_{0}(t)
$$

for each $t \in[0,1] \backslash \bigcup_{m=1}^{\infty} D_{1 / m}$. Hence the limit for $\longrightarrow_{S}$ is determined uniquely. Further, step functions are dense in $\mathbb{D}$ for the uniform topology and so for the constant sequence $x_{n} \equiv x_{0}$, $n=1,2, \ldots$, we have $x_{n} \longrightarrow_{S} x_{0}$. Since also a subsequence of a sequence convergent in the sense of $\longrightarrow_{S}$ is (obviously) $\longrightarrow_{S}$-convergent, we conclude that $\longrightarrow_{S}$ is an $\mathcal{L}$-convergence. It follows we have enough information to define a topology.

Definition 2.5 A set $F \subset \mathbb{D}$ is closed in $S$-topology, if it contains all limits of its $\longrightarrow_{S}$-convergent subsequences, i.e. if $x_{n} \in F, n=1,2, \ldots$, and $x_{n} \longrightarrow_{S} x_{0}$ then $x_{0} \in F$. The convergence of sequences in $S$-topology will be denoted by $\stackrel{*}{\longrightarrow} S$.

Remark 2.6 Similarly as in many other cases, $\stackrel{*}{\longrightarrow} S$, being an $\mathcal{L}^{*}$-convergence, may be weaker than the original $\mathcal{L}$-convergence $\longrightarrow_{S}$. This is not a real problem in view of the KantorovichKisyński recipe [9],[10]:

$x_{n} \stackrel{*}{\longrightarrow} S x_{0}$ if, and only if, in every subsequence $\left\{n_{k}\right\}$ one can find a further subsequence $\left\{n_{k_{l}}\right\}$ such that $x_{n_{k_{l}}} \longrightarrow x_{0}$.

In particular, relative $\stackrel{*}{\longrightarrow} S$-compactness of $K \subset \mathbb{D}$ and relative $\longrightarrow_{S}$-compactness of $K$ coincide, as well as sequential $\stackrel{*}{\longrightarrow} S$-continuity (三S-continuity) of $g: \mathbb{D} \rightarrow \mathbb{R}^{1}$ means the same as sequential $\longrightarrow_{S}$-continuity of $g$.

The reason for our interest in the $S$-topology is that (4) and (5) provide criteria of relative compactness for topology $S$. 
Lemma 2.7 If (4) and (5) hold for $K \subset \mathbb{D}$ then there exists a sequence $\left\{x_{n}\right\} \subset K$ and $x_{0} \in \mathbb{D}$ such that $x_{n} \longrightarrow_{S} x_{0}$.

Conversely, if in every sequence $\left\{x_{n}\right\} \subset K$ one can find a subsequence $\left\{x_{n_{k}}\right\}$ and $x_{0} \in \mathbb{D}$ such that $x_{n_{k}} \longrightarrow S x_{0}$, then $K$ satisfies both conditions (4) and (5).

Proof. Suppose (4) and (5) are satisfied for $K \subset \mathbb{D}$. Fix for the time being $\varepsilon>0$ and consider the map $v_{\varepsilon}(x)$ defined by (23). By Lemma 2.1

$$
\sup \left\{\left\|v_{\varepsilon}(x)\right\|(1): x \in K\right\}<+\infty,
$$

hence the set $\left\{v_{\varepsilon}(x): x \in K\right\}$ is a relatively $\longrightarrow_{w}$-compact subset of $\mathbb{V}$ and we can extract a sequence $\left\{x_{\varepsilon, n}\right\} \subset K$ such that $v_{\varepsilon}\left(x_{\varepsilon, n}\right) \longrightarrow_{w} v_{\varepsilon}$, for some $v_{\varepsilon} \in \mathbb{V}$.

Now let us set $\varepsilon=1,1 / 2,1 / 3, \ldots$ and apply the diagonal procedure in order to find a sequence $\left\{x_{n}\right\} \subset K$ such that for every $m \in \mathbb{N}$

$$
v_{1 / m}\left(x_{n}\right) \underset{w}{\longrightarrow} v_{1 / m}
$$

Let $\mathbb{Q} \subset[0,1]$ consist of those $t$ for which

$$
v_{1 / m}\left(x_{n}\right)(t) \rightarrow v_{1 / m}(t), \quad \text { as } n \rightarrow+\infty, m=1,2, \ldots
$$

We have $1 \in \mathbb{Q}$ and $[0,1] \backslash \mathbb{Q}$ is at most countable, hence $\mathbb{Q}$ is dense. In particular, for any $x \in \mathbb{D}$,

$$
\|x\|_{\infty}=\sup _{t \in \mathbb{Q}}|x(t)| .
$$

By $(34)$

$$
\begin{aligned}
\mid v_{1 / m}(t)- & v_{1 / k}(t) \mid \\
& =\lim _{n \rightarrow \infty}\left|v_{1 / m}\left(x_{n}\right)(t)-v_{1 / k}\left(x_{n}\right)(t)\right| \\
& \leq \limsup _{n \rightarrow \infty}\left|v_{1 / m}\left(x_{n}\right)(t)-x_{n}(t)\right|+\left|x_{n}(t)-v_{1 / k}\left(x_{n}\right)(t)\right| \\
& \leq \frac{1}{m}+\frac{1}{k}
\end{aligned}
$$

and by (35)

$$
\left\|v_{1 / m}-v_{1 / k}\right\|_{\infty} \leq \frac{1}{m}+\frac{1}{k}
$$

It follows that $v_{1 / m}$ uniformly converges to some $x_{0} \in \mathbb{D}$ and $\left\|x_{0}-v_{1 / m}\right\| \leq 1 / m$. Hence $x_{n} \longrightarrow_{S} x_{0}$.

The converse part follows immediately from Lemma 2.8 below.

Lemma 2.8 Suppose $x_{n} \stackrel{*}{\longrightarrow} x_{0}$. Then

$$
\begin{aligned}
\sup _{n \in \mathbb{N}}\left\|x_{n}\right\|_{\infty} & \leq C_{\infty}<+\infty, \\
\sup _{n \in \mathbb{N}} N^{a, b}\left(x_{n}\right) & \leq C^{a, b}<+\infty, a<b, a, b \in \mathbb{R}^{1}, \\
\sup _{n \in \mathbb{N}} N_{\eta}\left(x_{n}\right) & \leq C_{\eta}<+\infty, \eta>0, \\
\sup _{n \in \mathbb{N}}\left\|v_{\varepsilon}\left(x_{n}\right)\right\|(1) & \leq C_{\varepsilon}<+\infty, \varepsilon>0 .
\end{aligned}
$$


Proof. By (31) we may assume $x_{n} \longrightarrow_{S} x_{0}$. Because of inequalities (17), (18) and (19), it is enough to prove (39) only. But by the special way of construction of $v_{\varepsilon}(x)$, for any $y \in \mathbb{D}$ satisfying $\|y-x\|_{\infty} \leq \varepsilon / 3$, one has

$$
\|y\|(1) \geq \frac{1}{3}\left\|v_{\varepsilon}(x)\right\|(1)
$$

If $v_{n, \varepsilon / 3}$ is such that $\left\|x_{n}-v_{n, \varepsilon / 3}\right\|_{\infty} \leq \varepsilon / 3, n=0,1,2, \ldots$, and $v_{n, \varepsilon / 3} \longrightarrow_{w} v_{0, \varepsilon / 3}$, then

$$
+\infty>3 \sup _{n}\left\|v_{n, \varepsilon / 3}\right\|(1) \geq \sup _{n}\left\|v_{\varepsilon}\left(x_{n}\right)\right\|(1) .
$$

In Remark 2.4 we showed that $x_{n} \longrightarrow_{S} x_{0}$ implies pointwise convergence outside a countable set $D \subset[0,1)$. By $(31)$ we have

Corollary 2.9 If $x_{n} \stackrel{*}{\longrightarrow} S x_{0}$, then in each subsequence $\left\{x_{n_{k}}\right\}$ one can find a further subsequence $\left\{x_{n_{k_{l}}}\right\}$ and a countable set $D \subset[0,1)$ such that

$$
x_{n_{k_{l}}}(t) \longrightarrow x_{0}(t), \quad t \in[0,1] \backslash D .
$$

Given Corollary 2.9 we have lower semicontinuity of many useful functionals on $\mathbb{D}$.

Corollary 2.10 If $x_{n} \stackrel{*}{\rightarrow} x_{0}$ then:

$$
\begin{aligned}
\liminf _{n \in \mathbb{N}}\left\|x_{n}\right\|_{\infty} & \geq\left\|x_{0}\right\|_{\infty}, \\
\liminf _{n \in \mathbb{N}} N^{a, b}\left(x_{n}\right) & \geq N^{a, b}\left(x_{0}\right), \quad a<b, a, b \in \mathbb{R}^{1}, \\
\liminf _{n \in \mathbb{N}} N_{\eta}\left(x_{n}\right) & \geq N_{\eta}\left(x_{0}\right), \quad \eta>0, \\
\liminf _{n \in \mathbb{N}}\left\|v_{\varepsilon}\left(x_{n}\right)\right\|(1) & \geq\left\|v_{\varepsilon}\left(x_{0}\right)\right\|(1), \quad \varepsilon>0 .
\end{aligned}
$$

In view of (36) and (41) we have continuity for integral functionals.

Corollary 2.11 Let $\Phi:[0,1] \times \mathbb{R}^{1} \rightarrow \mathbb{R}^{1}$ be measurable and such that for each $t \in[0,1] \Phi(t, \cdot)$ is continuous and for each $C>0$

$$
\sup _{t \in[0,1]|x| \leq C} \sup _{|x|}|\Phi(t, x)|<+\infty .
$$

Let $\mu$ be an atomless finite measure on $[0,1]$. Then the mapping

$$
\mathbb{D} \ni x \mapsto \int_{[0,1]} \Phi(t, x(t)) d \mu(t) \in \mathbb{R}^{1}
$$

is S-continuous.

An important particular case is the Lebesgue measure on $[0,1]$ and

$$
\Phi(t, x)=\frac{1}{\delta} \mathbb{I}_{[u, u+\delta]}(t) \cdot x
$$


which gives the $S$-continuity of mappings

$$
\mathbb{D} \ni x \mapsto x_{u}^{\delta}=\frac{1}{\delta} \int_{[u, u+\delta]} x(t) d t .
$$

Since for $u<1$

$$
\lim _{\delta \searrow 0} x_{u}^{\delta}=x(u)=\pi_{u}(x)
$$

and $\pi_{1}$ is $S$-continuous, we conclude that Borel subsets of $(\mathbb{D}, S)$ coincide with $\sigma\left(\pi_{u}: u \in[0,1]\right)$. In addition, running $u$ and $\delta$ over rational numbers in $[0,1]$ we get a countable family of $S$-continuous functions which separate points in $\mathbb{D}$. In particular, any $S$-compact subset of $\mathbb{D}$ is metrisable. Another useful statement implied by continuity of $(48)$ is that $(\mathbb{D}, S)$ is a Hausdorff space.

Further, it follows from the very definition of the convergence $\longrightarrow_{S}$ that $x_{n} \longrightarrow_{S} x_{0}$ and $y_{n} \longrightarrow_{S} y_{0}$ implies $x_{n}+y_{n} \longrightarrow_{S} x_{0}+y_{0}$, i.e. addition is sequentially continuous and so from this point of view $S$-topology is nicer than the Skorohod's $J_{1}$ and $M_{1}$.

It should be, however, emphasized that contrary to Skorohod's topologies, the evaluations $\pi_{u}$ are for $u<1$ nowhere $S$-continuous. To see this, take $x=x_{0} \in \mathbb{D}$ and $0 \leq u<1$ and define

$$
x_{n}(t)=x_{0}(t)+\mathbb{I}_{[u, u+(1 / n))}(t) .
$$

Clearly, $x_{n} \longrightarrow_{S} x_{0}$, but $x_{n}(u)=x_{0}(u)+1 \not \rightarrow x_{0}(u)$.

Finally, let us notice that $S$ is not metrisable, as the following example shows.

Example 2.12 Let for $m, n \in \mathbb{N} x_{m, n}(t)=m \mathbb{1}_{[1 / 2,1 / 2+1 / n)}(t), t \in[0,1]$. Then for each fixed $m \in \mathbb{N}$ we have $x_{m, n} \longrightarrow_{S} 0, \quad$ as $n \rightarrow+\infty$. Let $n_{m} \rightarrow \infty$, as $m \rightarrow \infty$. The sequence $\left\{x_{m, n_{m}}\right\}_{m \in \mathbb{N}}$ does not contain any $\stackrel{*}{\longrightarrow} S$-convergent subsequence, for $\liminf \inf _{m \rightarrow \infty}\left\|x_{m, n_{m}}\right\|_{\infty}=+\infty$. And for any convergence generated by a metric there should exist a sequence $\left\{n_{m}\right\}$ such that $x_{m, n_{m}} \stackrel{*}{\longrightarrow} 0$.

We summarize all obtained results in

Theorem 2.13 The Skorohod space ID equipped with the sequential topology $S$ is a Hausdorff topological space which cannot be metricized. Moreover:

(i) There exists a countable family of $S$-continuous functions which separate points in $\mathbb{D}$.

(ii) Addition is sequentially continuous with respect to $\longrightarrow_{S}$.

(iii) Compact subsets $K \subset \mathbb{D}$ are metrisable.

(iv) A subset $K \subset \mathbb{D}$ is relatively $S$-compact if any of the equivalent sets of conditions (12)+(13), $(12)+(14)$ and $(15)+(16)$ is satisfied.

(v) $S$-Borel subsets $\mathcal{B}_{S}$ coincide with the standard $\sigma$-algebra generated by evaluations (projections).

(vi) $S$ is coarser (weaker) than the Skorohod's $J_{1}$ and $M_{1}$ topologies.

We conclude this section with two additional properties of the $S$-topology.

We have observed that the evaluations at $t<1$ are nowhere continuous. It is therefore interesting that the evaluations still can be used for identification of the limit. 
Proposition 2.14 Let $\mathbb{Q} \subset[0,1], 1 \in \mathbb{Q}$, be dense. Suppose $\left\{x_{n}\right\}$ is relatively $S$-compact and

$$
x_{n}(q) \rightarrow x_{0}(q), \quad \text { as } n \rightarrow+\infty, q \in \mathbb{Q} .
$$

Then $x_{n} \stackrel{*}{\longrightarrow} S x_{0}$.

Proof. Suppose along a subsequence $\left\{n_{k}\right\}$ we have $x_{n_{k}} \longrightarrow_{S} y_{0}$. Then for $q^{\prime}$ in some dense subset $\mathbb{Q}^{\prime} \subset[0,1), x_{n}\left(q^{\prime}\right) \rightarrow y_{0}\left(q^{\prime}\right)$. If $y_{0} \neq x_{0}$, then for some $\eta>0$ and $(u, v) \subset[0,1)$ one has $\left|y_{0}(t)-x_{0}(t)\right|>\eta$ for $t \in(u, v)$. Let $u<t_{1}<t_{2}<\ldots<v$ be such that:

1. $t_{1}, t_{3}, \ldots, t_{2 m-1}, \ldots \in \mathbb{Q}$

2. $t_{2}, t_{4}, \ldots, t_{2 m}, \ldots \in \mathbb{Q}^{\prime}$.

Then for each $m \in \mathbb{N} N_{\eta / 2}\left(x_{n_{k}}\right) \geq 2 m$ for $k$ large enough, hence $N_{\eta / 2}\left(x_{n_{k}}\right) \rightarrow+\infty$ and $\left\{x_{n_{k}}\right\}$ cannot be $S$-convergent. Hence $y_{0}=x_{0}$.

Clearly, not all topologies on $\mathbb{D}$ possess the property investigated in Proposition 2.14. For example, it is easy to find a sequence $\left\{x^{n}\right\} \subset \mathbb{D}$ which converges in measure to $x^{0} \equiv 0$ and is such that $x^{n}(q) \rightarrow 1$ for each rational $q \in[0,1]$. Hence Proposition 2.14 is not valid for the "pseudo-path" topology.

Another interesting feature of the $S$-topology is the continuity of the smoothing operation $\mathbb{D} \ni x \mapsto s_{\mu}(x) \in C\left([0,1]: \mathbb{R}^{1}\right)$, where

$$
s_{\mu}(x)(t)=\int_{0}^{t} x(s) d \mu(s)
$$

and $\mu$ is an atomless finite measure on $[0,1]$ (e.g. given by a density $p_{\mu}(s)$ ).

Proposition 2.15 The operation $s_{\mu}:(\mathbb{D}, S) \rightarrow\left(C,\|\cdot\|_{\infty}\right)$ is continuous.

Proof. Suppose $x_{n} \longrightarrow_{S} x_{0}$. Corollary 2.11 shows that for every $t \in[0,1] s_{\mu}\left(x_{n}\right)(t) \rightarrow s_{\mu}\left(x_{0}\right)(t)$. Hence only relative compactness of $\left\{s_{\mu}\left(x_{n}\right)\right\} \subset C\left([0,1]: \mathbb{R}^{1}\right)$ has to be verified. But this is straightforward:

$$
\sup _{n} \sup _{t<u<t+\delta}\left|s_{\mu}\left(x_{n}\right)(u)-s_{\mu}\left(x_{n}\right)(t)\right| \leq \sup _{n}\left\|x_{n}\right\|_{\infty} \times \sup _{t \in[0,1]} \mu([t, t+\delta]) \rightarrow 0,
$$

when $\delta \rightarrow 0$.

Remark 2.16 It is easy to see that the topology $\mathcal{S}$ can be defined also on the space $\mathbb{D}\left([0,1]: \mathbb{R}^{d}\right)$ of cádlág functions with values in finite dimensional space $\mathbb{R}^{d}$. It suffices either to define the convergence by coordinates or to consider in (28) convergence (26) of vector-valued measures and to restrict the attention in Lemma 2.1 to quantity $N_{\eta}$ only. An infinite dimensional generalization requires, however, more careful approach (e.g. in infinite dimension $\sigma$-additive vector valued measures may have infinite variation) and will not be considered here. 


\section{Convergence in distribution on $(\mathbb{D}, S)$}

In the previous section we checked the equality $\mathcal{B}_{S}=\sigma\left\{\pi_{t}: t \in[0,1]\right\}$. It follows that every probability measure on $\left(\mathbb{D}, \mathcal{B}_{S}\right)$ is tight. Let us denote the set of such measures by $\mathcal{P}(\mathbb{D})$. Further, the notions "random element in $\left(\mathbb{D}, \mathcal{B}_{S}\right)$ " and "stochastic process with trajectories in $\mathbb{D}$ " are synonymous and we see that the theory developed in Section 2 applies to usual objects.

It is a nice feature of Lemma 2.7 that we have

Proposition 3.1 Let $\left\{X_{\alpha}\right\}$ be a family of stochastic processes with trajectories in $\mathbb{D}$. The following statements are equivalent.

(i) $\left\{X_{\alpha}\right\}$ is uniformly S-tight.

(ii) $\left\{\left\|X_{\alpha}\right\|_{\infty}\right\}$ is a uniformly tight family as well as for each $a<b\left\{N^{a, b}\left(X_{\alpha}\right)\right\}$ is uniformly tight.

(iii) $\left\{\left\|X_{\alpha}\right\|_{\infty}\right\}$ is uniformly tight and for each $\eta>0\left\{N_{\eta}\left(X_{\alpha}\right)\right\}$ is uniformly tight.

(iv) For each $\varepsilon>0$ the family $\left\{\left\|v_{\varepsilon}\left(X_{\alpha}\right)\right\|(1)\right\}$ is uniformly tight (where $v_{\varepsilon}$ is defined by (23)).

And Theorem 1.1 gives us the direct Prohorov's theorem in $\mathcal{P}(\mathbb{D}, S)$ :

Theorem 3.2 Let $\left\{X_{\alpha}\right\}$ be a family of stochastic processes with trajectories in ID. If either of equivalent conditions (i)-(iv) in Proposition 3.1 is satisfied, then $\left\{X_{\alpha}\right\}$ is relatively compact.

The converse Prohorov's theorem, however, is not clear at all. And we are not going to investigate this question. Instead we shall equip $\mathcal{P}(\mathbb{D})$ with a sequential topology induced by $S$, which is finer than the weak-* topology, for which both the direct and the converse Prohorov's theorem hold and which is very close to the a.s. Skorohod representation. The advantage of such an approach is evident: we have effective tools (Prohorov's theorems, the a.s. Skorohod representation), more (in general) continuous functionals and if the converse Prohorov's theorem is valid for the weak-* topology, then the convergence of sequences in both topologies coincides (see [7]).

The advertized "new" convergence in distribution will be denoted by $\stackrel{*^{*}}{\longrightarrow} \mathcal{D}$ and $X_{n} \stackrel{*^{*}}{\longrightarrow} \mathcal{D} X_{0}$ will mean the following:

Definition 3.3 In every subsequence $\left\{X_{n_{k}}\right\}$ one can find a further subsequence $\left\{X_{n_{k_{l}}}\right\}$ and stochastic processes $\left\{Y_{l}\right\}$ defined on $\left([0,1], \mathcal{B}_{[0,1]}, \ell\right)$ such that

$$
Y_{l} \sim X_{n_{k_{l}}}, \quad l=1,2, \ldots
$$

for each $\omega \in[0,1]$

$$
Y_{l}(\omega) \underset{S}{\stackrel{*}{\longrightarrow}} Y_{0}(\omega), \quad \text { as } l \rightarrow \infty,
$$

and for each $\varepsilon>$ there exists an $S$-compact subset $K_{\varepsilon} \subset \mathbb{D}$ such that

$$
P\left(\left\{\omega \in[0,1]: Y_{l}(\omega) \in K_{\varepsilon}, l=1,2, \ldots\right\}\right)>1-\varepsilon .
$$


One can say that (52) and (53) describe "the almost sure convergence in compacts" and that (51), (52) and (53) define the strong a.s. Skorohod representation for subsequences ("strong" because of condition (53)).

We shall write $\mu_{n} \stackrel{*}{\Longrightarrow} \mu_{0}$ whenever $\mu_{n}=\mathcal{L}\left(X_{n}\right)$ and $X_{n} \stackrel{*^{\prime}}{\longrightarrow} X_{0}$.

It follows from Lemma 2.7 and Corollary 2.10 that on $(\mathbb{D}, S)$ there exists a countable family of lower semicontinuous functionals $\left\{\xi_{i}\right\}_{i \in \mathbb{I}}$ such that $K \subset \mathbb{D}$ is conditionally compact iff each $\xi_{i}$ is bounded on $K, i \in \mathbb{I I}$. Therefore we can apply Theorem 5.2 of [7] to obtain the direct and converse Prohorov's theorems for $\mathcal{O}_{S}(\stackrel{*}{\Longrightarrow})$.

Theorem 3.4 Let $\left\{X_{\alpha}\right\}$ be a family of stochastic processes with trajectories in $\mathbb{D}$. Then $\left\{X_{\alpha}\right\}$ is uniformly $S$-tight if and only if it is relatively compact with respect to " $\stackrel{*^{*}}{\longrightarrow}$ " on $(\mathbb{D}, S)$.

We have shown the topology $\mathcal{O}_{S}(\stackrel{*}{\Longrightarrow})$ induced by $S$ is as good as the weak-* topology on $\mathcal{P}\left(\mathbb{D}, J_{1}\right)$, of course, only from the point of view of proving limit theorems. Theorem 3.2 does not end the list of (formal) similarities between the mentioned topologies. We shall point three other properties of the topology $\mathcal{O}_{S}(\stackrel{*}{\Longrightarrow})$ which belong to standard tools of limit theory. It will be proved first that convergence of finite dimensional distributions can be used for identification of limits in $\stackrel{*}{\Longrightarrow}$-convergence (although the projections are nowhere continuous).

Theorem 3.5 Let $\mathbb{Q} \subset[0,1]$ be dense, $1 \in \mathbb{Q}$. Suppose that for each finite subset $\mathbb{Q}_{0}=\left\{q_{1}<q_{2}<\right.$ $\left.\ldots<q_{m}\right\} \subset \mathbb{Q}$ we have as $n \rightarrow \infty$

$$
\left(X_{n}\left(q_{1}\right), X_{n}\left(q_{2}\right), \ldots, X_{n}\left(q_{m}\right)\right) \underset{\mathcal{D}}{\longrightarrow}\left(X_{0}\left(q_{1}\right), X_{0}\left(q_{2}\right), \ldots, X_{0}\left(q_{m}\right)\right)
$$

where $X_{0}$ is a stochastic process with trajectories in $\mathbb{D}$.

If $\left\{X_{n}\right\}$ is relatively compact with respect to $\stackrel{*^{*}}{\longrightarrow} \mathcal{D}$, then $X_{n} \stackrel{*^{*}}{\longrightarrow} \mathcal{D} X_{0}$.

Proof. Let $\psi_{0}(x)=\|x\|_{\infty}$ and let $\psi_{i}(x)=N_{1 / i}(x), i=1,2, \ldots$. Define also $\Psi(x)=\left(\psi_{i}(x)\right)_{i=0,1,2, \ldots}$ $\in \mathbb{R}^{\infty}$ and $\Phi(x)=(x(q))_{q \in \mathbb{Q}} \in \mathbb{R}^{\mathbb{Q}}$.

By Theorem $3.2\left\{\Psi\left(X_{n}\right)\right\}$ is uniformly tight in $\mathbb{R}^{\infty}$. Hence at least along some subsequence we have in $\mathbb{R}^{\infty} \times \mathbb{R}^{\mathbb{Q}}$

$$
\left(\Phi\left(X_{n}\right), \Psi\left(X_{n}\right)\right) \underset{\mathcal{D}}{\longrightarrow}\left(\Phi\left(X_{0}\right), Z\right) .
$$

By Skorohod's theorem on the a.s. representation there exist random elements $\left(U_{n}, V_{n}\right), n=$ $0,1,2, \ldots$, defined on $\left([0,1], \mathcal{B}_{[0,1]}, \ell\right)$ and such that

$$
\begin{aligned}
\left(U_{n}, V_{n}\right) & \sim\left(\Phi\left(X_{n}\right), \Psi\left(X_{n}\right)\right), \quad n=1,2, \ldots \\
\left(U_{0}, V_{0}\right) & \sim\left(\Phi\left(X_{0}\right), Z\right)
\end{aligned}
$$

and for each $\omega \in[0,1]$

$$
U_{n}(\omega) \rightarrow U_{0}(\omega) \text { in } \mathbb{R}^{\infty}, V_{n}(\omega) \rightarrow V_{0}(\omega) \text { in } \mathbb{R}^{\mathbb{Q}} .
$$

We claim that

there exists a measurable mapping $\Theta: \mathbb{R}^{\infty} \rightarrow \mathbb{D}$ such that $\Theta\left(U_{n}\right) \sim X_{n}, n=$ $0,1,2, \ldots$, and $V_{n}=\Psi \circ \Theta\left(U_{n}\right), \ell$-a.s., $n=1,2, \ldots$ 
$\Phi$ is a measurable and one-to-one mapping from $\mathbb{D}$ into $\mathbb{R}^{\infty}$. We know $S$-compact subsets of $\mathbb{D}$ are metrisable, hence $\Phi$ maps each $S$-compact subset $K$ onto a Borel subset $\Phi(K)$ of $\mathbb{R}^{\infty}$. The same holds also for any $\sigma$-compact subset of $\mathbb{D}$. Since laws of all $X_{0}, X_{1}, X_{2}, \ldots$ are $S$-tight, we can find a common $\sigma$-compact support $K_{0}$, i.e. $P\left(X_{n} \in K_{0}\right)=1, n=0,1,2, \ldots$ If we set $\Theta(y)=\Phi^{-1}(y)$ for $y \in \Phi\left(K_{0}\right)$ and $\Theta(y)=0$ outside $\Phi\left(K_{0}\right)$, then $X_{n}=\Theta\left(\Phi\left(X_{n}\right)\right)$ almost surely on the probability space where $X_{n}$ is defined and $U_{n}=\Phi\left(\Theta\left(U_{n}\right)\right)$ l-a.s. We have also $\Psi\left(X_{n}\right)=\Psi\left(\Theta\left(\Phi\left(X_{n}\right)\right)\right)$ almost surely and so $V_{n}=\Psi\left(\Theta\left(U_{n}\right)\right)$ l-a.s. by $(56)$.

Choose $\omega \in[0,1]$ in a "good" subset of full measure and consider $\left\{x_{n}=\Theta\left(U_{n}(\omega)\right)\right\}_{n \in \mathbb{N}} \subset \mathbb{D}$. We have $x_{n}(q) \rightarrow x_{0}(q), q \in \mathbb{Q}$, and for each $i=0,1,2, \ldots \psi_{i}\left(x_{n}\right) \rightarrow\left(V_{0}(\omega)\right)_{i}$, hence $\sup _{n} \psi_{i}\left(x_{n}\right)<$ $+\infty$. By Proposition $2.14 x_{n} \stackrel{*}{\longrightarrow} S x_{0}$. Hence $\Theta\left(U_{n}\right)$ is the a.s. Skorohod representation for $X_{n}$ (in fact, for a subsequence of $X_{n}$ ) and so $X_{n} \stackrel{*}{\longrightarrow} \mathcal{D} X_{0}$.

Only a slightly modified proof give us

Theorem 3.6 Let $\mathbb{Q} \subset[0,1]$ be dense, $1 \in \mathbb{Q}$. Suppose $\left\{X_{n}\right\}$ is a uniformly $S$-tight sequence and that for each finite sequence $q_{1}<q_{2}<\ldots<q_{m}$ of elements of $\mathbb{Q}$ we have

$$
\left.\left(X_{n}\left(q_{1}\right), X_{n}\left(q_{2}\right), \ldots, X_{n}\left(q_{m}\right)\right) \underset{\mathcal{D}}{\longrightarrow} \tilde{\nu}_{\left(q_{1}, q_{2}, \ldots, q_{m}\right)}\right)
$$

where $\tilde{\nu}_{\left(q_{1}, q_{2}, \ldots, q_{m}\right)}$ is a probability distribution on $\mathbb{R}^{m}$. Then for each finite sequence $t_{1}<t_{2}<$ $\ldots<t_{m}$ and each approximating sequence $q_{1, l}<q_{2, l}<\ldots<q_{m, l}, q_{j, l} \searrow t_{j}$, as $l \rightarrow \infty,\left(q_{m, l}=\right.$ 1 if $\left.t_{m}=1\right)$, probability distributions $\tilde{\nu}_{\left(q_{1, l}, q_{2, l}, \ldots, q_{m, l}\right)}$ weakly converge to some limit $\nu_{\left(t_{1}, t_{2}, \ldots, t_{m}\right)}$. Moreover, there is a stochastic process $X_{0}$ with trajectories in $\mathbb{D}$ such that for every finite sequence $t_{1}<t_{2}<\ldots<t_{m}$

$$
\left(X_{0}\left(t_{1}\right), X_{0}\left(t_{2}\right), \ldots, X_{0}\left(t_{m}\right)\right) \sim \nu_{\left(t_{1}, t_{2}, \ldots, t_{m}\right)},
$$

and $X_{n_{k}} \stackrel{*}{\longrightarrow} \mathcal{D} X_{0}$.

Remark 3.7 The Skorohod $J_{1}$-topology also possesses the properties described in Theorems 3.5 and 3.6 (see $[20]$ ).

An especially convenient tool for investigations of the topology $\mathcal{O}_{S}(\stackrel{*}{\Longrightarrow})$ is provided by the following decomposition.

Theorem 3.8 A family $\left\{X_{\alpha}\right\}$ of stochastic processes with trajectories in $\mathbb{D}$ is uniformly $S$-tight if, and only if, for each $\varepsilon>0$ we can decompose processes $X_{\alpha}$ in the following way:

$$
X_{\alpha}(t)=R_{\alpha, \varepsilon}(t)+U_{\alpha, \varepsilon}(t)-V_{\alpha, \varepsilon}(t), \quad t \in[0,1],
$$

where all processes $R_{\alpha, \varepsilon}, U_{\alpha, \varepsilon}$ and $V_{\alpha, \varepsilon}$ are adapted to the natural right-continuous filtration generated by $X_{\alpha}$, trajectories of $R_{\alpha, \varepsilon}$ are uniformly small:

$$
\left\|R_{\alpha, \varepsilon}\right\|_{\infty} \leq \varepsilon
$$

$U_{\alpha, \varepsilon}$ and $V_{\alpha, \varepsilon}$ are nonnegative and nondecreasing (i.e. have paths in $\mathbb{V}^{+}$) and both $\left\{U_{\alpha, \varepsilon}(1)\right\}$ and $\left\{V_{\alpha, \varepsilon}(1)\right\}$ are uniformly tight families of random variables. 
Proof. By Theorem 3.6, the family $\left\{X_{\alpha}\right\}$ is uniformly $S$-tight iff for each $\varepsilon>0$ the family of random variables $\left\{\left\|v_{\varepsilon}\left(X_{\alpha}\right)\right\|(1)\right\}$ is uniformly tight, where processes $v_{\varepsilon}\left(X_{\alpha}\right)(t)$ are defined by (23) and are adapted by Corollary 2.2. Hence it is enough to set $R_{\alpha}(t)=X_{\alpha}(t)-v_{\varepsilon}\left(X_{\alpha}\right)(t)$ and decompose $v_{\varepsilon}\left(X_{\alpha}\right)(t)$ into a difference of two increasing processes $U_{\alpha, \varepsilon}(t)$ and $V_{\alpha, \varepsilon}(t)$ in such a way that

$$
\left\|v_{\varepsilon}\left(X_{\alpha}\right)\right\|(1)=U_{\alpha, \varepsilon}(1)+V_{\alpha, \varepsilon}(1)
$$

Remark 3.9 It follows from (62) that any fact on increasing processes may contribute to the knowledge of $S$-topology. The very close relation between the weak topology on $\mathbb{V}^{+}$and the $S$ topology on $\mathbb{D}$ becomes clear, if we realize that, similarly to $M_{1}$, the topology $S$ induces on $\mathbb{V}^{+}$ exactly the topology of weak convergence (notice, however, that on $\mathbb{V}=\mathbb{V}^{+}-\mathbb{V}^{+}$the topology $S$ is coarser than the weak topology, while $M_{1}$ is incompatible with the latter!). So we can consider the $S$-topology as a natural extension on $\mathbb{D}$ of the notion of weak convergence of elements of $\mathbb{V}^{+}$.

Theorem 3.10 Let $\left\{V_{\alpha}\right\}$ be a family of stochastic processes with trajectories in $\mathbb{V}^{+}$. Suppose that $\left\{V_{\alpha}(1)\right\}$ is uniformly tight. Then there exists a sequence $\left\{V_{n}\right\} \subset\left\{V_{\alpha}\right\}$, an increasing process $V_{0}$ and a countable subset $D \subset[0,1)$ such that for all finite sets $\mathbb{Q}_{0}=\left\{q_{1}<q_{1}<\ldots<q_{m}\right\} \subset[0,1] \backslash D$

$$
\left(V_{n}\left(q_{1}\right), V_{n}\left(q_{2}\right), \ldots, V_{n}\left(q_{m}\right)\right) \underset{\mathcal{D}}{\longrightarrow}\left(V_{0}\left(q_{1}\right), V_{0}\left(q_{2}\right), \ldots, V_{0}\left(q_{m}\right)\right)
$$

Proof. Let $\mu_{\alpha}$ be a random measure on $\left([0,1], \mathcal{B}_{[0,1]}\right)$ given by formula

$$
\mu_{\alpha}([0, t], \omega)=\frac{V_{\alpha}(t, \omega)}{1+V_{\alpha}(1, \omega)}, \quad t \in[0,1] .
$$

Since $\mu_{\alpha}$ takes values in the space $\mathcal{M}_{\leq 1}=\mathcal{M}_{\leq 1}([0,1])$ of measures on compact space $[0,1]$ with total mass smaller than 1 , we can extract a sequence $\mu_{n}$ such that on the space $\mathcal{M}_{\leq 1} \times \mathbb{R}^{+}$

$$
\left(\mu_{n}, V_{n}(1)\right) \underset{\mathcal{D}}{\longrightarrow}\left(\mu_{0}, Z_{0}\right)
$$

Since $\mathcal{M}_{\leq 1} \times \mathbb{R}^{+}$is a Polish space we may assume without loss of generality that almost surely

$$
\mu_{n}(\cdot, \omega) \Longrightarrow \mu_{0}(\cdot, \omega), \quad V_{n}(1, \omega) \rightarrow Z_{0}(\omega)
$$

Let us consider the mapping $[0,1] \ni t \mapsto E \mu_{0}([0, t], \omega)=\left(E \mu_{0}\right)([0, t])$. Outside a countable set $D \subset[0,1)$ we have $\left(E \mu_{0}\right)(\{t\})=0$, hence $\mu_{0}(\{t\}, \omega)=0$ for almost all $\omega$, and so by $(67)$ and for $t \notin D$

$$
\mu_{n}([0, t], \omega) \rightarrow \mu_{0}([0, t], \omega)
$$

or

$$
V_{n}(t, \omega) \rightarrow\left(1+Z_{0}(\omega)\right) \mu_{0}([0, t], \omega)=: V_{0}(t, \omega) .
$$

The theorem has been proved.

Now we are ready to prove a corresponding result for $(\mathbb{D}, S)$ which (in some sense) improves Theorem 5 of [14], where finite dimensional convergence outside a set of Lebesgue measure 0 was obtained (but only convergence in distribution with respect to the "pseudo-path topology" was assumed). 
Theorem 3.11 Let $\left\{X_{\alpha}\right\}$ be a uniformly $S$-tight family of stochastic processes with trajectories in $\mathbb{D}$. Then there exists a sequence $\left\{X_{n}\right\} \subset\left\{X_{\alpha}\right\}$, a process $X_{0}$ with trajectories in $\mathbb{D}$ and $a$ countable subset $D \subset[0,1)$ such that for all finite sets $\mathbb{Q}_{0}=\left\{q_{1}<q_{1}<\ldots<q_{m}\right\} \subset[0,1] \backslash D$

$$
\left(X_{n}\left(q_{1}\right), X_{n}\left(q_{2}\right), \ldots, X_{n}\left(q_{m}\right)\right) \underset{\mathcal{D}}{\longrightarrow}\left(X_{0}\left(q_{1}\right), X_{0}\left(q_{2}\right), \ldots, X_{0}\left(q_{m}\right)\right) .
$$

In particular, $X_{n} \stackrel{*^{*}}{\longrightarrow} \mathcal{D} X_{0}$.

Proof. According to (62), for each $m \in \mathbb{I}$ we can decompose

$$
X_{\alpha}=R_{\alpha, 1 / m}+U_{\alpha, 1 / m}-V_{\alpha, 1 / m}
$$

with $U_{\alpha, 1 / m}$ and $V_{\alpha, 1 / m}$ uniformly tight on $\left(\mathbb{V}^{+}, S\right)$. By tightness assumptions one can find a subsequence such that on the space $(\mathbb{D}, S) \times\left(\mathbb{V}^{+}, S\right)^{\mathbb{N}}$

$$
\begin{aligned}
\left(X_{n}, U_{n, 1}, V_{n, 1}, \ldots,\right. & \left.U_{n, 1 / m}, V_{n, 1 / m}, \ldots\right) \\
& \stackrel{*}{\mathcal{D}}\left(X_{0}, U_{1}, V_{1}, \ldots, U_{m}, V_{m}, \ldots\right) .
\end{aligned}
$$

It means that passing again to a subsequence we have the a.s. Skorohod representation, i.e. without loss of generality we may assume that

$$
\begin{aligned}
& X_{n}(\omega) \underset{S}{\stackrel{*}{\longrightarrow}} X_{0}(\omega) \text { in } \mathbb{D}, \\
& U_{n, 1 / m}(\omega) \stackrel{*}{\stackrel{\leftrightarrow}{\longrightarrow}} U_{m}(\omega) \text { in } \mathbb{V}^{+}, \quad m \in \mathbb{N}, \\
& V_{n, 1 / m}(\omega) \stackrel{*}{\stackrel{*}{\longrightarrow}} V_{m}(\omega) \text { in } \mathbb{V}^{+}, \quad m \in \mathbb{N} \text {. }
\end{aligned}
$$

Since $X_{n}(\omega) \stackrel{*}{\longrightarrow} S X_{0}(\omega)$ and because of $(62)$ we have

$$
\left\|U_{m}(\omega)-V_{m}(\omega)-X_{0}(\omega)\right\|_{\infty} \leq 2 / m
$$

and so for every finite subset $\left\{t_{1}<t_{2}<\ldots<t_{r}\right\} \subset[0,1]$ we have as $m \rightarrow \infty$

$$
\begin{aligned}
\left(U_{m}\left(t_{1}\right)-V_{m}\left(t_{1}\right), U_{m}\left(t_{2}\right)-V_{m}\left(t_{2}\right)\right. & \left., \ldots, U_{m}\left(t_{r}\right)-V_{m}\left(t_{r}\right)\right) \\
& \longrightarrow \mathcal{D} \quad\left(X_{0}\left(t_{1}\right), X_{0}\left(t_{2}\right), \ldots, X_{0}\left(t_{r}\right)\right) .
\end{aligned}
$$

On the other hand, applying Theorem 3.10 we see that as $n \rightarrow \infty$

$$
\begin{aligned}
\left(U_{n, 1 / m}\left(q_{1}\right), V_{n, 1 / m}\left(q_{1}\right), U_{n, 1 / m}\left(q_{2}\right), V_{n, 1 / m}\left(q_{2}\right), \ldots, U_{n, 1 / m}\left(q_{r}\right), V_{n, 1 / m}\left(q_{r}\right)\right) \\
\longrightarrow \mathcal{D} \quad\left(U_{m}\left(q_{1}\right), V_{m}\left(q_{1}\right), U_{m}\left(q_{2}\right), V_{m}\left(q_{2}\right), \ldots, U_{m}\left(q_{r}\right), V_{m}\left(q_{r}\right)\right)
\end{aligned}
$$

for every finite subset $\mathbb{Q}_{0}=\left\{q_{1}<q_{2}<\ldots<q_{r}\right\} \subset[0,1]$ outside a countable subset $D_{m} \subset[0,1)$. Hence outside $D=D_{1} \cup D_{2} \cup \ldots$ we have (70).

Remark 3.12 In Introduction we stated that we do not know whether $S$ is a regular topological space. Except for very special cases (like metrisability or existence of a countable fundamental system of compact sets - what is not the case) regularity is not easy to verify within the class 
of sequential spaces. One thing requires special attention. We have checked that addition is sequentially continuous, i.e. continuous with respect to the natural sequential topology on the product ("convergence on coordinates"). It does not mean continuity as a function on the product $\mathbb{D} \times \mathbb{D}$ with the product topology $S \times S$, i.e. the coarsest topology with respect to which both projections are continuous. It is known that the product topology on the product of linear sequential spaces is again sequential if one of the spaces is e.g. locally compact, what is not true again. (see [2], p. 213, for details). Therefore $(\mathbb{D}, S)$ is not a linear topological space.

Some comments are relevant.

- Due to the a.s. Skorohod representation given by Theorem 1.1 only sequential continuity of addition is required by possible applications. Hence the last problem is unimportant.

- If $(\mathbb{D}, S)$ were completely regular, we could repeat the reasoning given after Theorem 3.2.

- It seems to be possible to develop the theory within the standard framework and define a completely regular topology $\tau$, for which $S$ is the sequential topology generated by $\tau$ convergence of sequences. But then we encounter all difficulties proper to the theory of weak convergence on nonmetric spaces: lack of the a.s. Skorohod's representation, local character of the direct Prohorov's theorem, etc. In addition, if we use the direct Prohorov's theorem, then we enter again the present framework. Problems of this type are in details described in [7] in the case of infinite dimensional separable Hilbert space equipped with the weak topology.

\section{Uniform $S$-tightness and semimartingales}

Theorem 3.2 provides several sets of conditions equivalent to uniform $S$-tightness. Inequalities (18) and (19) suggest the easiest way of proving uniform $S$-tightness: one has to check whether families $\left\{\left\|X_{\alpha}\right\|_{\infty}\right\}$ and $\left\{N^{a, b}\left(X_{\alpha}\right)\right\}$, for each $a<b$, are bounded in probability. For example, if $X$ is a supermartingale, then one can use the classical Doob's inequalities (see [1], Ch. VI):

$$
\begin{gathered}
P\left(\sup _{t \in[0,1]}|X(t)| \geq \lambda\right) \leq 3 \lambda^{-1} \sup _{t \in[0,1]} E|X(t)|, \\
E N^{a, b}(X) \leq \frac{1}{b-a}\left(|a|+\sup _{t \in[0,1]} E|X(t)|\right) .
\end{gathered}
$$

It follows immediately that any sequence $\left\{X_{n}\right\}$ of supermartingales satisfying

$$
\sup _{n} \sup _{t \in[0,1]} E\left|X_{n}(t)\right|<+\infty
$$

is uniformly $S$-tight. The most general result of such kind concerns semimartingales and belongs to Stricker [19]. We shall restate it using terminology of the paper [8] and the setting of $S$-topology.

Let $\left\{X_{\alpha}\right\}$ be a family of stochastic processes with trajectories in $\mathbb{D}$, with $X_{\alpha}$ defined on the stochastic basis $\left(\Omega^{\alpha}, \mathcal{F}^{\alpha},\left\{\mathcal{F}_{t}^{\alpha}\right\}_{t \in[0,1]}, P^{\alpha}\right)$ and adapted to filtration $\left\{\mathcal{F}_{t}^{\alpha}\right\}_{t \in[0,1]}$. We say that Condition $U T$ holds for $\left\{X_{\alpha}\right\}$, if the family of elementary stochastic integrals $\left\{\int H_{-}^{\alpha} d X_{\alpha}(1)\right\}$ with integrands bounded by 1 is uniformly tight. (By an elementary stochastic integral with integrands 
bounded by 1 we mean random variable of the form

$$
\sum_{i=1}^{m} H^{\alpha}\left(t_{i-1}\right)\left(X_{\alpha}\left(t_{i}\right)-X_{\alpha}\left(t_{i-1}\right)\right),
$$

where $m \in \mathbb{N}, 0=t_{0}<t_{1}<\ldots<t_{m}=T, H^{\alpha}\left(t_{i}\right) \leq 1$ and $H^{\alpha}\left(t_{i}\right)$ is $\mathcal{F}^{\alpha}\left(t_{i}\right)$-measurable for $i=0,1, \ldots, m$. Condition UT was considered for the first time in [19]. The reader may find conditions which follow the line of (73) and are sufficient for Condition UT in [8]. For equivalent reformulations in terms of predictable characteristics we refer to [13]. Here we stress the fact that the family consisting of a single process $X$ satisfies Condition UT if, and only if, $X$ is a semimartingale (see e.g. [15]). Therefore in what follows we shall deal with semimartingales only.

The essential step in the proof of Theorem 2 of [19] gives us

Theorem 4.1 Condition UT implies uniform S-tightness.

By Theorem 3.11 any set $\left\{X_{\alpha}\right\}$ of semimartingales satisfying Condition UT contains a sequence $X_{n} \stackrel{*}{\rightarrow} \mathcal{D} X_{0}$ for which finite dimensional convergence holds outside a countable set $D \subset[0,1)$ : for every finite subset $\left\{0 \leq q_{1}<q_{2}<\ldots<q_{m} \leq 1\right\} \subset \mathbb{Q}=[0,1] \backslash D$

$$
\left(X^{n}\left(q_{1}\right), X^{n}\left(q_{2}\right), \ldots, X^{n}\left(q_{m}\right)\right) \underset{\mathcal{D}}{\longrightarrow}\left(X^{0}\left(q_{1}\right), X^{0}\left(q_{2}\right), \ldots, X^{0}\left(q_{m}\right)\right)
$$

on the space $\mathbb{R}^{m}$. If (74) is satisfied for every finite subset $\left\{0 \leq q_{1}<q_{2}<\ldots<q_{m} \leq 1\right\}$ of some set $\mathbb{Q}$, we shall write $X_{n} \longrightarrow_{\mathcal{D}_{f}(\mathbb{Q})} X_{0}$.

Since $\mathbb{Q}=[0,1] \backslash D$ is dense in $[0,1]$ we can apply Lemma 1.3 of [8] and conclude that $X_{0}$ is a semimartingale. Let us observe that the pseudo-path topology gives the finite dimensional convergence over a set of full Lebesgue measure only and so our methods give an improvement of Theorem 2 in [19].

Theorem 4.2 Suppose Condition UT holds for a family of semimartingales $\left\{X_{\alpha}\right\}$. Then there exists a sequence $\left\{X_{n}\right\} \subset\left\{X_{\alpha}\right\}$ and a semimartingale $X_{0}$ such that

$$
X_{n} \underset{\mathcal{D}}{\stackrel{*}{\longrightarrow}} X_{0} \text { and } X_{n} \underset{\mathcal{D}_{f}(\mathbb{Q})}{\longrightarrow} X_{0},
$$

where $1 \in \mathbb{Q}$ and the complement of $\mathbb{Q}$ in $[0,1]$ is at most countable.

Under somewhat different assumptions, and using completely different methods, a result similar to Theorem 4.2 has been obtained in [11] (Theorem 1.1).

The two subsequent results are based on Theorems 3.5 and 3.6 and are not valid for the pseudopath topology.

Theorem 4.3 Suppose $\mathbb{Q} \subset[0,1]$ is dense, $1 \in \mathbb{Q}$ and $X_{n} \longrightarrow_{\mathcal{D}_{f}(\mathbb{Q})} X_{0}$, where $X_{0}$ has trajectories in $\mathbb{I D}$.

If Condition UT is satisfied for $\left\{X_{n}\right\}$, then $X_{0}$ is a semimartingale and $X_{n} \stackrel{{ }^{*}}{\longrightarrow} \mathcal{D} X_{0}$. 
Theorem 4.4 Let $\mathbb{Q}$ be as in Theorem 4.3. Suppose for each finite sequence $q_{1}<q_{2}<\ldots<q_{m}$ of elements of $\mathbb{Q}$ we have

$$
\left(X_{n}\left(q_{1}\right), X_{n}\left(q_{2}\right), \ldots, X_{n}\left(q_{m}\right)\right) \underset{\mathcal{D}}{\longrightarrow} \tilde{\nu}_{\left(q_{1}, q_{2}, \ldots, q_{m}\right)}
$$

where $\tilde{\nu}_{\left(q_{1}, q_{2}, \ldots, q_{m}\right)}$ is a probability distribution on $\mathbb{R}^{m}$.

If Condition UT holds for $\left\{X_{n}\right\}$, then there exists a semimartingale $X_{0}$ such that $X_{n} \stackrel{*}{\longrightarrow} \mathcal{D}$ $X_{0}$. Moreover, for each finite sequence $t_{1}<t_{2}<\ldots<t_{m}$ and each approximating sequence $q_{1, l}<q_{2, l}<\ldots<q_{m, l}, q_{j, l} \searrow t_{j}$, as $l \rightarrow \infty,\left(q_{m, l}=1\right.$ if $\left.t_{m}=1\right)$, probability distributions $\tilde{\nu}_{\left(q_{1, l}, q_{2, l}, \ldots, q_{m, l}\right)}$ weakly converge to the joint distribution of $\left(X_{0}\left(q_{1}\right), X_{0}\left(q_{2}\right), \ldots, X_{0}\left(q_{m}\right)\right)$.

Eventually, let us notice that the topology $S$ arises in a quite natural manner in limit theorems for the Ito stochastic integrals (see [6]).

\section{References}

[1] Dellacherie, C., Meyer, P.A. (1980) Probabilités et potentiel, Chapters V-VIII, Hermann, Paris 1980.

[2] Engelking, R., General Topology, Helderman, Berlin 1989.

[3] Fernique, X., Processus linéaires, processus généralisés, Ann. Inst. Fourier (Grenoble), 17 (1967), 1-92.

[4] Fernique, X., Convergence en loi de variables alétoires et de fonctions alétoires, propriétes de compacité des lois, II, in: J. Azéma, P.A. Meyer, M. Yor, (Eds.) Séminaire de Probabilités XXVII, Lecture Notes in Math., 1557, 216-232, Springer, Berlin 1993.

[5] Jakubowski, A., The a.s. Skorohod representation for subsequences in nonmetric spaces, Theory Probab. Appl., 42 (1997), 209-216.

[6] Jakubowski, A., Convergence in various topologies for stochastic integrals driven by semimartingales, Ann. Probab., 24 (1996), 2141-2153.

[7] Jakubowski, A., From convergence of functions to convergence of stochastic processes. On Skorokhod's sequential approach to convergence in distribution., to appear in A Volume in Honour of A.V. Skorokhod, VSP, 1997.

[8] Jakubowski, A., Mémin, J., Pages, G., Convergence en loi des suites d'intégrales stochastiques sur l'espace $\mathbb{I}^{1}$ de Skorokhod, Probab. Th. Rel. Fields, 81 (1989), 111-137.

[9] Kantorowich, L.V., Vulih, B.Z. \& Pinsker, A.G., Functional Analysis in Partially Ordered Spaces (in Russian), Gostekhizdat, Moscow 1950.

[10] Kisyński, J., Convergence du type L, Colloq. Math., 7 (1960), 205-211.

[11] Kurtz, T., Random time changes and convergence in distribution under the Meyer-Zheng conditions, Ann. Probab., 19 (1991), 1010-1034. 
[12] Kurtz, T., Protter, P., Weak limit theorems for stochastic integrals and stochastic differential equations, Ann. Probab., 19 (1991), 1035-1070.

[13] Mémin, J., Słomiński, L. Condition UT et stabilité en loi des solutions d'equations différentieles stochastiques, Sém. de Probab. XXV, Lecture Notes in Math., 1485, 162-177, Springer, Berlin 1991.

[14] Meyer, P.A., Zheng, W.A., Tightness criteria for laws of semimartingales, Ann. Inst. Henri Poincaré B, 20 (1984), 353-372.

[15] Protter, Ph., Stochastic Integration and Differential Equations. A New Approach., 2nd Ed., Springer 1992.

[16] Skorohod,A.V., Limit theorems for stochastic processes, Theor. Probability Appl., 1 (1956), 261-290.

[17] Słomiński, L., Stability of strong solutions of stochastic differential equations, Stoch. Proc. Appl., 31 (1989), 173-202.

[18] Słomiński, L., Stability of stochastic differential equations driven by general semimartingales, Dissertationes Math., CCCXLIX (1996), 113 p.

[19] Stricker, C., Lois de semimartingales et critères de compacité, Séminares de probabilités XIX. Lect. Notes in Math., 1123, Springer, Berlin 1985.

[20] Topsøe, F., A criterion for weak convergence of measures with an application to convergence of measures on $\mathbb{D}[0,1]$, Math. Scand., 25 (1969), 97-104. 